\title{
络合法制备均匀 $\gamma-\mathrm{Fe}_{2} \mathrm{O}_{3}$ 纳米颗粒@多层石墨烯复合材料研究
}

\author{
邱小贞，徐军明，徐桢，宋开新，武军，应智花，胡炜薇
}

(杭州电子科技大学电子信息学院, 杭州 310018)

摘 要: 采用水热反应中的金属离子络合一步制备均匀超细磁性 $\gamma-\mathrm{Fe}_{2} \mathrm{O}_{3}$ 纳米颗粒@多层石墨烯复合材料, 无需对 石墨烯进行氧化处理。采用超声法制备多层石墨烯作为基片，制备方法简单，石墨烯表面的含氧官能团少。以 $\mathrm{FeCl}_{2}$ 为反应物，以 $\mathrm{DMF}(\mathrm{N}, \mathrm{N}$ 二甲基甲酰胺)和水混合液作为溶剂，其中 $\mathrm{DMF}$ 能起到络合金属离子的作用。实验研究了 乙酸钠、反应温度及填充度对制备产物的影响。采用 $\mathrm{X}$ 射线衍射 $\mathrm{XRD}) 、 \mathrm{X}$ 射线光电子能谱 $(\mathrm{XPS}) 、$ 扫描电子显微 镜(SEM)、透射电子显微镜(TEM)对复合材料进行微结构分析, 采用振动样品磁强计(VSM)测试了复合材料的磁性 能。研究结果表明: 利用亚铁离子与 DMF 形成的络合物与碳环的 $\pi-\pi$ 吸附作用可以在多层石墨烯表面生成铁氧化 物。通过控制亚铁离子的氧化速度和氧化铁的生长速度, 在多层石墨烯表面获得了尺寸小于 $10 \mathrm{~nm}$ 的均匀 $\gamma-\mathrm{Fe}_{2} \mathrm{O}_{3}$ 纳米颗粒, 复合材料具有良好的磁性能。

关 键 词: 纳米颗粒; 磁性三氧化二铁; 石墨烯; 水热法; 复合材料

中图分类号: TQ174

文献标识码: A

\section{Preparation of Uniform Magnetic $\mathrm{Fe}_{2} \mathrm{O}_{3}$ Nanoparticles@multi-layer Graphene Composites with Complexation Method}

\author{
QIU Xiao-Zhen, XU Jun-Ming, XU Zhen, SONG Kai-Xin, WU Jun, YING Zhi-Hua, HU Wei-Wei \\ (College of Electronic Information, Hangzhou Dianzi University, Hangzhou 310018, China)
}

\begin{abstract}
One-step hydrothermal reaction and complexation method was used to prepare the extra uniform $\gamma-\mathrm{Fe}_{2} \mathrm{O}_{3}$ nanoparticles@multi-layer graphene, which was unnecessary to introduce oxygen function group on graphene. Multi-layer graphene was prepared by simple sonication method that will not introduce rich oxygen functional groups on its surface. Reagent $\mathrm{FeCl}_{2}$, mixture solvent DMF and water were used for hydrothermal reaction, in which DMF could form complexion with metal ion. The effects of sodium acetate, reaction temperature and filling ratio on the prepared product were studied. X-ray diffraction(XRD), X-ray photoelectron spectroscope(XPS), scanning electron microscope (SEM), and transmission electron microscope (TEM) were used to analyze the microstructures of the composite materials. The magnetic properties of the composites were measured by vibrating sample magnetometer (VSM). The results show that $\pi-\pi$ reaction between carbon ring and complexation of $\mathrm{Fe}^{2+}$ with DMF can be used to prepare iron oxide on the multi-layer graphene. Homogeneous $\gamma-\mathrm{Fe}_{2} \mathrm{O}_{3}$ nanoparticles with size less than $10 \mathrm{~nm}$ can be prepared through the control of oxidation speed of $\mathrm{Fe}^{2+}$ and growth velocity of iron oxide, with good magnetic properties.
\end{abstract}

Key words: nanoparticles; $\gamma$ - $\mathrm{Fe}_{2} \mathrm{O}_{3}$; graphene; hydrothermal; composite

收稿日期: 2015-12-21; 收到修改稿日期：2016-01-27

基金项目：浙江省自然科学基金(LY16E020009); 国家自然科学基金(61376005); 浙江省 “电子科学与技术” 重中之重学科开放基金 Natural Science Foundation of Zhejiang Province (LY16E020009); National Natural Science Foundation of China (61376005); "Electronic Science and Technology" _ Z Zhejiang Open Foundation of the Most Important Subjects

作者简介：邱小贞(1989-), 女, 硕士研究生. E-mail: 805973141@qq.com

通讯作者：徐军明, 副教授. E-mail: xujunming@hdu.edu.cn 
磁性铁氧化物 $\mathrm{Fe}_{3} \mathrm{O}_{4}$ 和 $\gamma-\mathrm{Fe}_{2} \mathrm{O}_{3}$ 纳米颗粒在生 物 $^{[1-3]}$ 、医学 ${ }^{[4-5]}$ 、储能 ${ }^{[6-10]}$ 等领域得到了广泛研究。 通过与石墨烯复合可以解决纳米材料的团聚问题, 并且发挥两者的优势 $[1-5,7-8,10-18]$ 。在石墨烯表面获 取分布均匀、尺寸小且直径分布窄的纳米氧化物颗 粒一直是研究的重点。目前, 大部分研究主要采用 氧化石墨烯(GO)基底来制备磁性氧化物纳米颗粒 复合材料 ${ }^{[1,4,7,10]}$, 其制备过程是通过氧官能团的作 用吸附金属离子, 并通过加热氧化成氧化物纳米颗 粒。但氧化石墨烯碳环破坏严重, 导电性能下降严重, 并且制备的纳米颗粒均匀性还有待提高。在制备过 程中或制备完成后对 $\mathrm{GO}$ 进行还原处理可以在一定 程度上恢复氧化石墨烯的导电性 ${ }^{[3,5,8,11-19]}$ 。Dai 等 ${ }^{[20]}$ 采用两步法在微氧化的石墨烯上制备了复合材料。 而在完全没有进行氧化处理的石墨烯表面制备金属 氧化物纳米颗粒一直是复合材料制备的难点。

多层石墨烯(ML-GNS) ${ }^{[21]}$ 制备方法简单, 表面 含氧官能团少, 其电性能及比表面积等特性能满足 一定要求, 可用于探索非氧化石墨烯表面制备金属 氧化物的科学问题。我们研发了一种通过有机分子 络合金属离子的方法制备 $\mathrm{Co}_{3} \mathrm{O}_{4}$ 石墨烯复合材料的 方法 ${ }^{[21]}$ 。本研究采用该方法制备超细且均匀分布的 磁性 $\gamma-\mathrm{Fe}_{2} \mathrm{O}_{3}$ 纳米粒子, 为磁性纳米铁氧化物/石墨 烯复合材料的应用提供基础研究。

\section{1 实验方法}

\section{1 原材料}

膨胀石墨(Graphite), N,N-二甲基甲酰胺(DMF, 分析纯), 蒸馏水(自制), 四水合氯化亚铁(分析纯), 无水乙酸钠(分析纯)。

\section{2 复合材料的制备}

$\operatorname{DMF}(8 \mathrm{~mL})$ 和蒸馏水 $(2 \mathrm{~mL})$ 形成混合溶剂, 加入 膨胀石墨 $(20 \mathrm{mg})$ 后超声 $3 \mathrm{~h}$ 制得多层石墨烯溶液 ${ }^{[21]}$ 。 在溶液中加入 $100 \mathrm{mg}$ 的氯化亚铁和不同含量的乙酸 钠, 磁力搅拌 $5 \mathrm{~min}$ 后将混合液倒入聚四氟乙烯内祄 的水热反应釜中, 在一定温度下保温 $3 \mathrm{~h}$, 冷却后采用 蒸馏水和酒精各清洗 3 次, 取出沉淀物在 $60^{\circ} \mathrm{C}$ 下烘干, 即可得到铁氧化物@石墨烯复合材料。

为了研究乙酸纳对产物的影响, 乙酸钠添加量分 别为 $0 、 50 、 150 、 200 \mathrm{mg}$ 的样品, 进行填充度为 $35 \%$, 保温温度为 $120^{\circ} \mathrm{C}$ 的处理, 样品分别标记为 NaAc-0、

NaAc-50、NaAc-150、NaAc-200。为了研究温度对产 物的影响, 保持乙酸钠添加量为 $200 \mathrm{mg}$, 填充度为 $35 \%$, 进行 $130^{\circ} \mathrm{C} 、 120^{\circ} \mathrm{C} 、 110^{\circ} \mathrm{C} 、 100^{\circ} \mathrm{C}$ 的试验, 样
品分别标记为 $T-130 、 T-120 、 T-110 、 T-100$ 。为了研 究水热罐填充度对产物的影响, 对 $200 \mathrm{mg}$ 乙酸钠添 加量, $120{ }^{\circ} \mathrm{C}$ 反应的样品在水热罐中进行填充度为 $20 \% 、 30 \% 、 40 \% 、 50 \%$ 反应，其样品标记为: FR-20、

FR-30、FR-40、FR-50。

\section{3 复合材料微结构表征和磁性能测试}

采用 X 射线衍射仪(XRD, Rigaku Ultima IV, 铜 靶, $40 \mathrm{kV}, 30 \mathrm{~mA}$ )进行物相分析; 采用 $\mathrm{X}$ 射线光电 子能谱仪(XPS, ThermoESCALab 250iX)进行元素价 态分析; 采用扫描电子显微镜(SEM, Hitachi S-4800) 和透射电子显微镜(TEM, Philips 2130)观察表面形 貌和微结构; 采用振动样品磁强计 (VSM, Lakeshore7407)对样品的磁性能进行分析。

\section{2 结果和分析}

\section{1 样品的物相分析}

图 1 是样品的 XRD 图谱, 图中 $2 \theta=26.4^{\circ}$ 和 $54.5^{\circ}$ 处的特征峰对应多层石墨烯的(002)及(004)晶面。图 1(A)显示乙酸钠对产物的影响。当水热反应中不添加 乙酸钠时, 产物中形成的物相是 $\alpha-\mathrm{Fe}_{2} \mathrm{O}_{3}$ (PDF 24-0072); 当添加乙酸钠 $50 \mathrm{mg}$ 时, 反应产物中出现 $\gamma-\mathrm{Fe}_{2} \mathrm{O}_{3}$ 衍射峰(PDF 39-1346)。随着乙酸钠添加量的增 加, 产物中 $\alpha-\mathrm{Fe}_{2} \mathrm{O}_{3}$ 的衍射峰逐渐降低, 而 $\gamma-\mathrm{Fe}_{2} \mathrm{O}_{3}$ 的 衍射峰相对增强, 说明 $\gamma-\mathrm{Fe}_{2} \mathrm{O}_{3}$ 在产物中的含量逐渐 增加。当乙酸钠添加量为 150 和 $200 \mathrm{mg}$ 时, 主要成分 是 $\gamma-\mathrm{Fe}_{2} \mathrm{O}_{3}$, 但仍存在 $\alpha-\mathrm{Fe}_{2} \mathrm{O}_{3}$ 衍射峰。进一步提高乙 酸钠添加量时, 发现始终存在少量 $\alpha-\mathrm{Fe}_{2} \mathrm{O}_{3}$ 。

当保持乙酸钠为 $200 \mathrm{mg}$, 改变水热反应的温度获 得的样品的 XRD 图谱如图 1(B)所示。通过比较 $\alpha-\mathrm{Fe}_{2} \mathrm{O}_{3}$ 相的最强峰和 $\gamma-\mathrm{Fe}_{2} \mathrm{O}_{3}$ 相的最强峰可以看出, 随着反应 温度的降低, $\gamma-\mathrm{Fe}_{2} \mathrm{O}_{3}$ 相的衍射峰增强。当反应温度为 $100^{\circ} \mathrm{C}$ 时, $\alpha-\mathrm{Fe}_{2} \mathrm{O}_{3}$ 相在 XRD 图谱中几乎不显示。经谢 乐公式计算, $\gamma-\mathrm{Fe}_{2} \mathrm{O}_{3}$ 相的晶粒尺寸在 $9.3 \mathrm{~nm}$ 左右。由 于 XRD 图谱无法直接区分 $\gamma-\mathrm{Fe}_{2} \mathrm{O}_{3}$ 和 $\mathrm{Fe}_{3} \mathrm{O}_{4}$ 相, 因此, 对 T-100 样品进行了 XPS 表征, 如图 2 所示。图 2(A) 的全谱扫描显示样品中存在 $\mathrm{Fe} 、 \mathrm{C} 、 \mathrm{O}$ 峰, 说明样品没 有引入杂质。图 2(B)是 $\mathrm{Fe} 2 \mathrm{p}$ 谱峰, $\mathrm{Fe} 2 \mathrm{p}_{3 / 2}$ 的位置在 $710.7 \mathrm{eV}, \mathrm{Fe} 2 \mathrm{p}_{1 / 2}$ 的位置在 $724.4 \mathrm{eV}$, 并且在 $719 \mathrm{eV}$ 处 有个明显的卫星峰, 与报道的 $\gamma-\mathrm{Fe}_{2} \mathrm{O}_{3}$ 图谱相近 ${ }^{[22]}$ 。

图 1(C)显示了反应物填充度由 $20 \%$ 增大到 $50 \%$ 获得的产物的 XRD 图谱(保持乙酸钠为 $200 \mathrm{mg}$, 水 热反应温度为 $120^{\circ} \mathrm{C}$ )。可以看到, 当填充度为 $20 \%$ 时, 产物为单一的晶相 $\alpha-\mathrm{Fe}_{2} \mathrm{O}_{3}$ 。随着填充度的增加, $\gamma-\mathrm{Fe}_{2} \mathrm{O}_{3}$ 相对量增加, 当填充度为 $40 \%$ 和 $50 \%$ 时, 产 

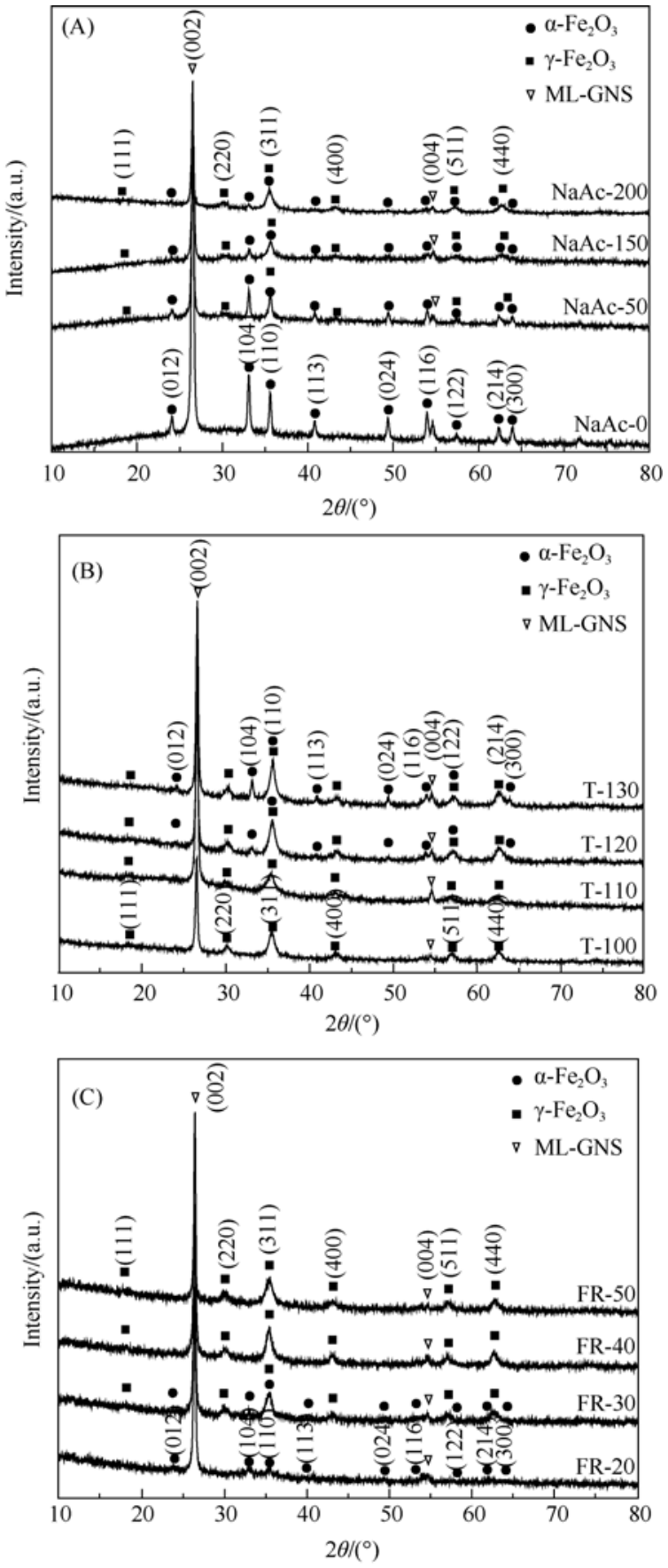

图 1 样品的 XRD 图谱

Fig. 1 XRD patterns of as-prepared samples Influence of (A) sodium acetate addition, (B) reaction temperature and (C) filling ratio

物为 $\gamma-\mathrm{Fe}_{2} \mathrm{O}_{3}$ 单相。

\section{2 样品的形貌观察}

图 3 是 NaAc-0 样品的 SEM 照片, 当反应液中 不添加乙酸钠时, 反应得到的是颗粒尺寸较大的 $\alpha-\mathrm{Fe}_{2} \mathrm{O}_{3}$, 其尺寸在亚微米大小, 尺寸均匀性差, 分 布较稀，具有明显的团聚现象。

图 4(A)是 NaAc-200 样品的 SEM 照片, 图 4(C) 和 4(D)是 NaAc-200 样品的 TEM 照片。相比没有添 加乙酸钠的样品, 添加乙酸钠后石墨烯表面的氧化物
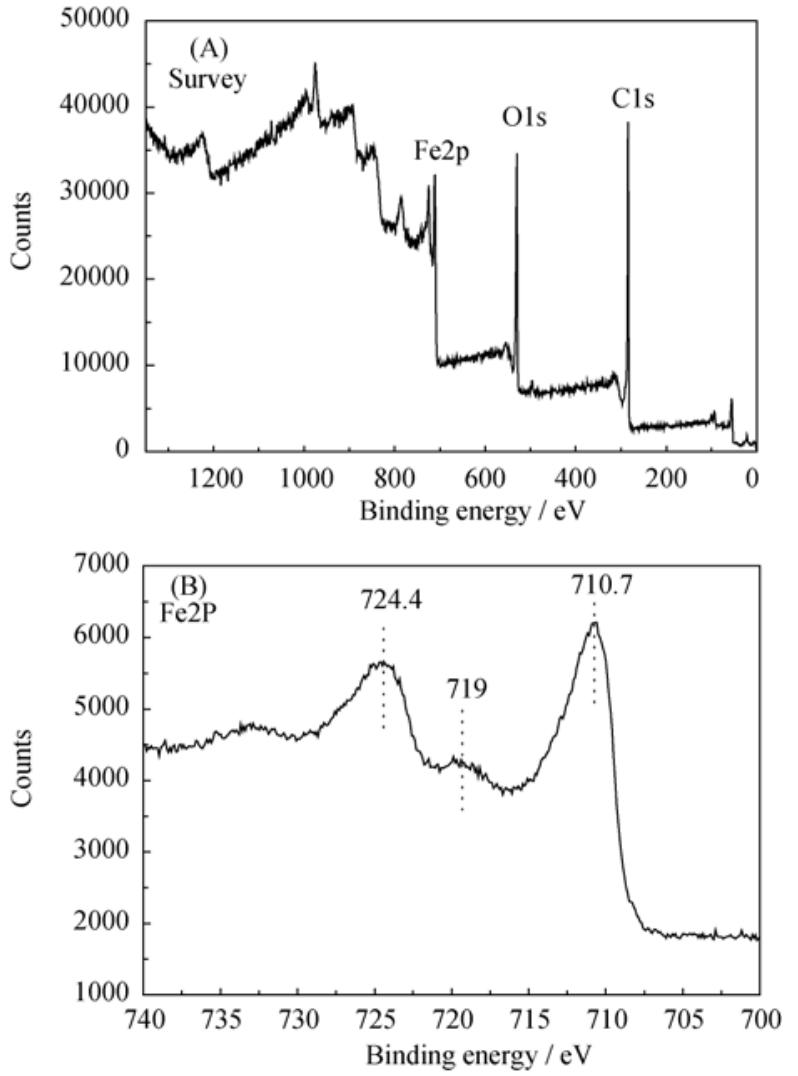

图 2 T-100 样品的 XPS 图谱

Fig. 2 XPS of T-100 sample

(A) Survey; (B) Fe2p spectrum

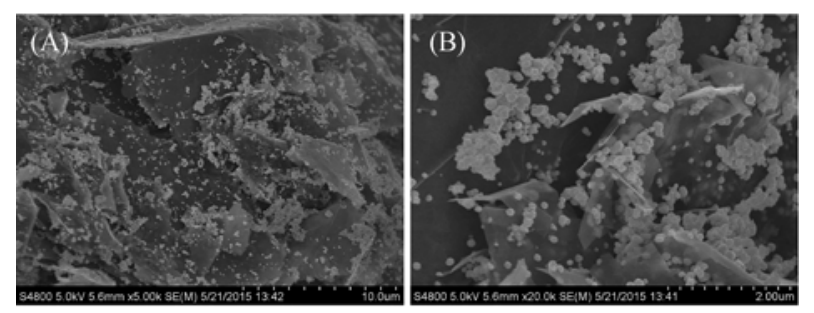

图 $3 \mathrm{NaAc}-0$ 样品的 SEM 照片

Fig. 3 SEM images of the NaAc-0 sample

(A) Low magnification; (B) High magnification

颗粒尺寸减小, 分布均匀性提高。但还是存在着少 量的大颗粒及部分的团聚现象, 如图 4(A)和 4(C)中 圆形区域所示。图 4(D) 是对图 4(C) 方框中的小颗粒 区域的放大图，可以看到该部分纳米颗粒分布均匀, 尺寸在 $15 \mathrm{~nm}$ 以下。当水热反应温度上升到 $130^{\circ} \mathrm{C}$, 其微观结构与 T-120 基本相同, 如图 4(B)。

图 5 是水热反应温度降低到 $100^{\circ} \mathrm{C}$, 即 $\mathrm{T}-100$ 样 品的 SEM 和 TEM 照片, 从图中可以看到, 该温度制 备的样品已基本没有出现大颗粒，除了极少部分发 生团聚外, 主要是由颗粒度很小的 $\gamma-\mathrm{Fe}_{2} \mathrm{O}_{3}$ 纳米颗粒 组成。从图 5(A)、(B)中可以看到, 这些纳米颗粒在 石墨烯片上基本形成连续膜。透射电镜图 5(D)显示, 这 些纳米颗粒尺寸大小均匀, 分布均匀, 粒径在 $10 \mathrm{~nm}$ 以 下，在石墨烯表面的分布密度高。 

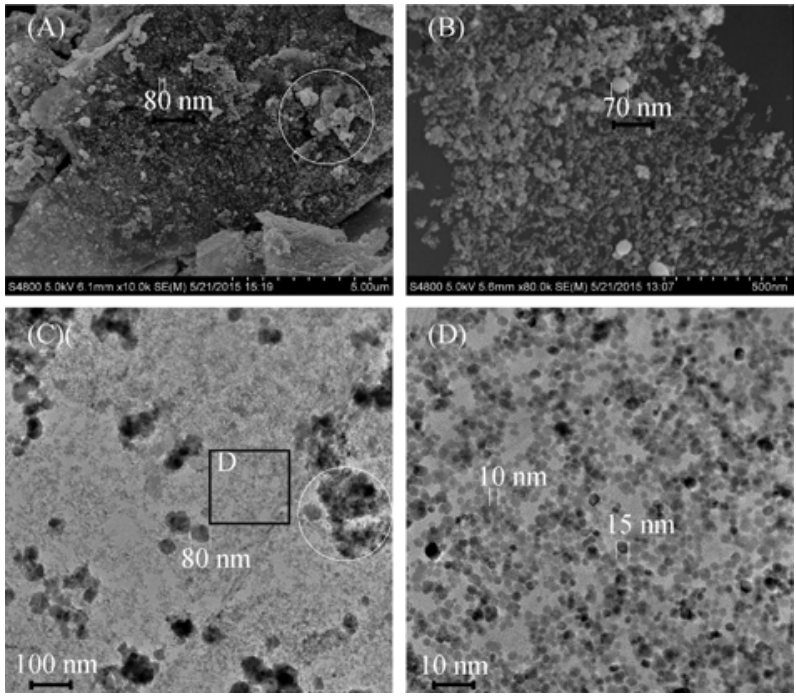

图 4 具有 $\gamma-\mathrm{Fe}_{2} \mathrm{O}_{3}$ 与 $\alpha-\mathrm{Fe}_{2} \mathrm{O}_{3}$ 混合相产物的 SEM 和 TEM 照片

Fig. 4 SEM and TEM images $\gamma-\mathrm{Fe}_{2} \mathrm{O}_{3}$ and $\alpha-\mathrm{Fe}_{2} \mathrm{O}_{3}$ mixes products

(A) SEM image of the NaAc-200 sample; (B) SEM image of the T-130 sample; (C) Low magnification TEM image of the NaAc-200 sample; (D) Enlargement of the part shown in figure (C)
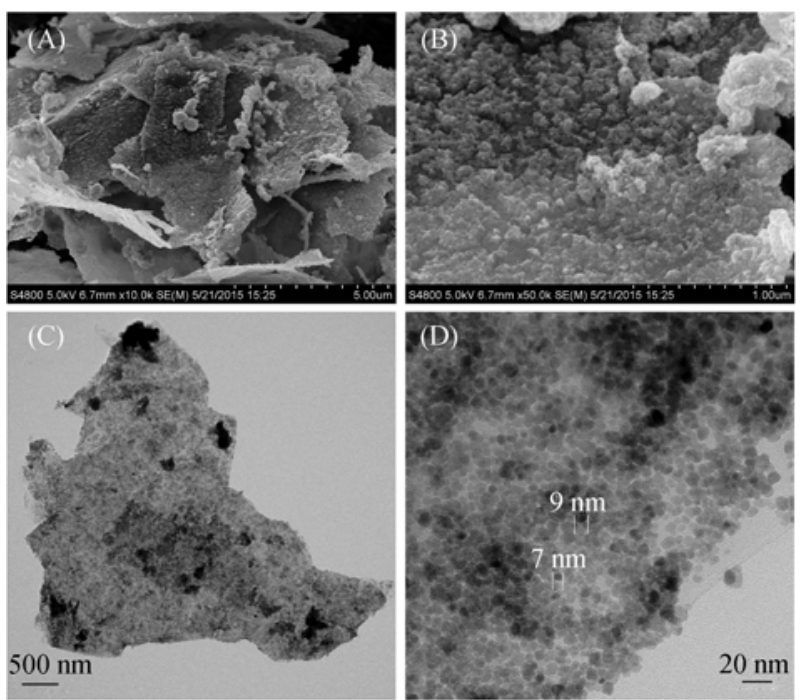

图 5 具有 $\gamma-\mathrm{Fe}_{2} \mathrm{O}_{3}$ 单相产物的 SEM 和 TEM 照片

Fig. 5 SEM and TEM images of the single-phase $\gamma-\mathrm{Fe}_{2} \mathrm{O}_{3}$ product (SEM images of the T-100 sample at low (A) and high (B) magnification; TEM images of the T-100 sample at low (C) ang high (D) magnification)

图 6 是纳米粒子的高分辨 TEM 照片, $0.253 \mathrm{~nm}$ 晶面间距对应 $\gamma-\mathrm{Fe}_{2} \mathrm{O}_{3}$ 的(311)晶面, $0.296 \mathrm{~nm}$ 晶面间 距对应 $\gamma-\mathrm{Fe}_{2} \mathrm{O}_{3}(220)$ 晶面。同时, 可以观察到单个纳 米颗粒是由一个晶粒组成。

\section{3 分析讨论}

膨胀石墨超声制备的多层石墨烯表面不会引入 大量的含氧官能团 ${ }^{[21]}$ 。因此, 石墨烯与金属氧化物 纳米颗粒的复合主要不是依靠氧的价键结合。络合 法制备复合材料的过程如图 7 所示。DMF 与水的混 合溶剂使石墨烯分散良好, 并与亚铁离子产生络合

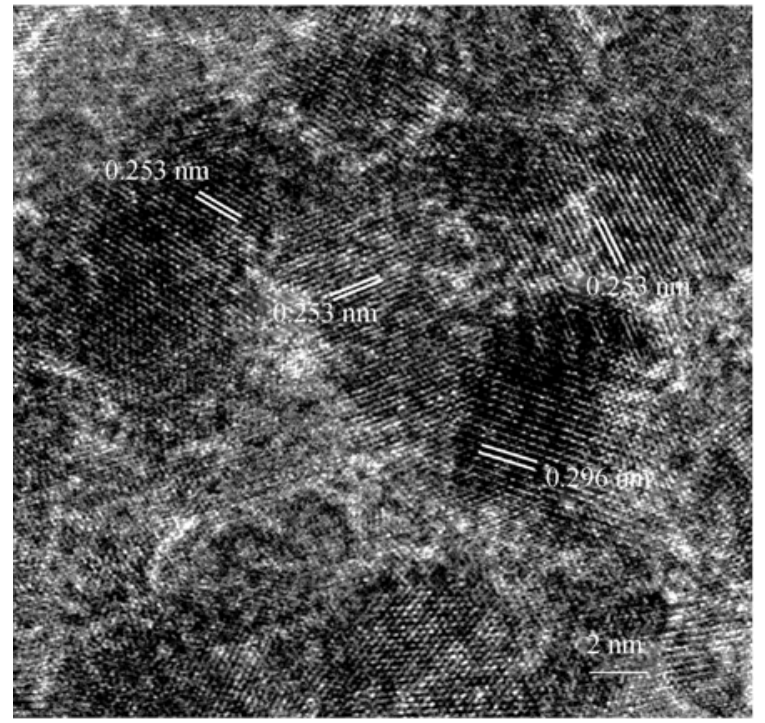

图 $6 \mathrm{~T}-100$ 样品的高分辨透射电镜图

Fig. 6 High resolution TEM image for T-100 sample

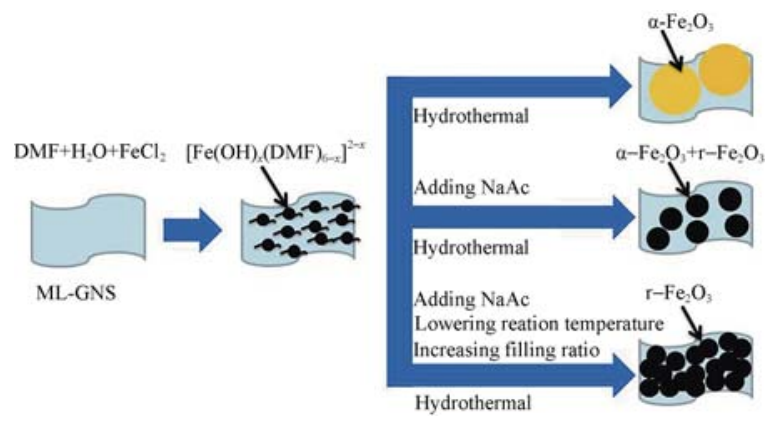

图 7 制备过程示意图

Fig. 7 Schematic diagram of formation process

形成 $\left(\left[\mathrm{Fe}(\mathrm{OH})_{x}(\mathrm{DMF})_{6-x}\right]^{2-x}\right)^{[23]}$, 络合物与石墨烯产 生 $\pi-\pi$ 作用而被吸附到石墨烯表面 ${ }^{[21]}$ 。在水热条件 下 $\left[\mathrm{Fe}(\mathrm{OH})_{x}(\mathrm{DMF})_{6-x}\right]^{2-x}$ 分解成氢氧化亚铁, 氢氧化 亚铁随后氧化成氧化铁。 $\gamma-\mathrm{Fe}_{2} \mathrm{O}_{3}$ 是反尖晶石结构, 可通过进一步氧化 $\mathrm{Fe}_{3} \mathrm{O}_{4}$ 获得 ${ }^{[24]}$, 因此能够获取 $\mathrm{Fe}_{3} \mathrm{O}_{4}$ 是形成 $\gamma-\mathrm{Fe}_{2} \mathrm{O}_{3}$ 的关键。而 $\mathrm{Fe}_{3} \mathrm{O}_{4}$ 的形成需要 在溶液中同时存在 $\mathrm{Fe}^{2+}$ 和 $\mathrm{Fe}^{3+}$, 因此, $\mathrm{Fe}^{2+}$ 离子的氧 化速度不能太快。

当没有添加醋酸钠时, 溶液的氧化能力强, 亚 铁离子被快速氧化成三价铁, 氧化铁的生长速度快, 从而得到晶粒较大的 $\alpha-\mathrm{Fe}_{2} \mathrm{O}_{3}$ 。添加醋酸钠后, 铁氧 化物的团聚和颗粒尺寸明显减小, 并使 $\alpha-\mathrm{Fe}_{2} \mathrm{O}_{3}$ 向 $\gamma-\mathrm{Fe}_{2} \mathrm{O}_{3}$ 转变, 这主要是由于乙酸根离子会吸附于铁 离子表面，抑制铁离子的氧化及抑制氧化物纳米颗 粒的长大 ${ }^{[21]}$ 。另外，依赖于 DMF 在水热条件下具有 的一定还原能力 ${ }^{[25-26]}$, 使二价铁离子和三价铁离子 同时存在, 从而能得到 $\mathrm{Fe}_{3} \mathrm{O}_{4}$, 而 $\mathrm{Fe}_{3} \mathrm{O}_{4}$ 在长时间氧 的作用下逐渐被氧化为 $\gamma-\mathrm{Fe}_{2} \mathrm{O}_{3}$ 。但该种情况下仍有 部分生长成 $\alpha-\mathrm{Fe}_{2} \mathrm{O}$ 。因此, 最终获得 $\alpha-\mathrm{Fe}_{2} \mathrm{O}_{3}$ 和 $\gamma-\mathrm{Fe}_{2} \mathrm{O}_{3}$ 混合纳米颗粒。 
降低水热反应温度或增大水热反应填充度, 可 以进一步减小溶液的氧化能力, 减小氧化物的生长 速度, 从而获得单一的 $\gamma-\mathrm{Fe}_{2} \mathrm{O}_{3}$ 纳米颗粒。反应温度 的降低还有利于减小纳米颗粒的尺寸和氧化物纳米 颗粒的团聚。

\section{4 样品的 VSM 分析}

石墨烯引入缺陷后可以获得一定的弱磁性 ${ }^{[27]}$, 而与磁性纳米颗粒复合可以获得更好的磁性能。图 8 是 $\alpha-\mathrm{Fe}_{2} \mathrm{O}_{3}\left(\mathrm{NaAc}-0\right.$ 样品)、 $\alpha-\mathrm{Fe}_{2} \mathrm{O}_{3}$ 与 $\gamma-\mathrm{Fe}_{2} \mathrm{O}_{3}$ 混合 颗粒(T-120 样品)及 $\gamma-\mathrm{Fe}_{2} \mathrm{O}_{3}$ (T-100 样品)三种复合材 料的磁滞回线，它们的比饱和磁化强度分别为 2.6、 44.6 和 $50.5 \mathrm{~A} \cdot \mathrm{m}^{2} / \mathrm{kg}$, 而矫顽力分别为 $20.17 、 7.39$ 、 $4.49 \mathrm{kA} / \mathrm{m}$ 。本研究采用的多层石墨烯比报道所用的 单层石墨烯具有更少的表面。并且制备的纳米颗粒 直径更小, 纳米材料界面增多引起的比饱和磁化强 度会下降得更大。即便如此，与已报道的纳米 $\mathrm{Fe}_{3} \mathrm{O}_{4} /$ 氧化石墨烯复合材料 ${ }^{[14,15,28-30]}$ 磁性能基本相 当。良好的磁性能主要来源于良好的结晶性和 $\gamma-\mathrm{Fe}_{2} \mathrm{O}_{3}$ 颗粒高分布密度。

\section{3 结论}

采用络合法通过水热反应一步制备了铁纳米氧 化物@石墨烯复合材料，无需对石墨烯进行氧化处 理。该方法主要依靠 DMF 分子与亚铁离子产生络 合后与石墨烯产生 $\pi-\pi$ 吸附而在石墨烯表面沉积, 在水热条件下形成铁氧化物纳米颗粒。没有添加乙 酸钠的情况下，反应产物为 $\alpha-\mathrm{Fe}_{2} \mathrm{O}_{3}$, 颗粒不均匀且 尺寸较大。随着乙酸钠添加量的增多，反应产物逐 渐由 $\alpha-\mathrm{Fe}_{2} \mathrm{O}_{3}$ 转变为 $\alpha-\mathrm{Fe}_{2} \mathrm{O}_{3}$ 和 $\gamma-\mathrm{Fe}_{2} \mathrm{O}_{3}$ 的混合物。 通过降低制备温度、提高填充度可以抑制亚铁离子 的被氧化程度，从而获得均匀的尺寸小于 $10 \mathrm{~nm}$ 的

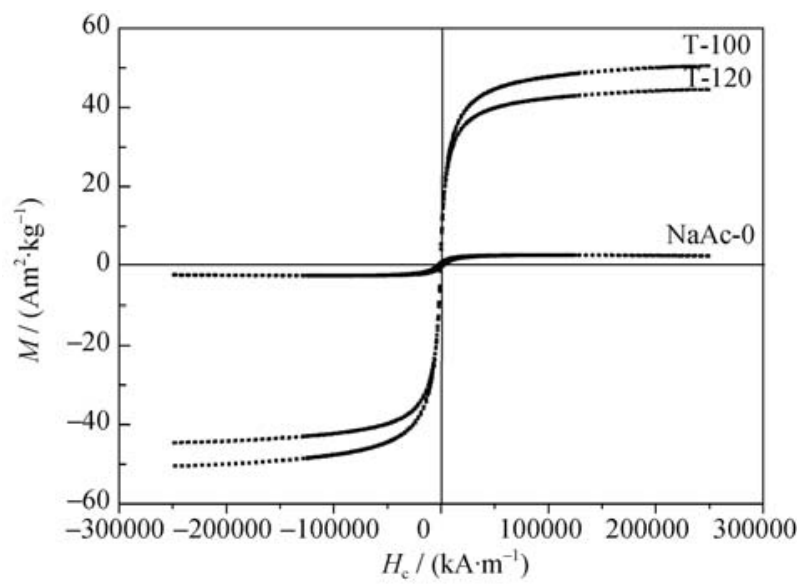

图 8 样品的磁滞回线图

Fig. 8 Hysteresis loop diagram of samples
$\gamma-\mathrm{Fe}_{2} \mathrm{O}_{3}$ 单相纳米颗粒。经 VSM 测试，制备的 $\gamma-\mathrm{Fe}_{2} \mathrm{O}_{3}$ 纳米颗粒@多层石墨烯具有高的饱和磁感 应强度，具有良好的软磁特性。

\section{参考文献:}

[1] XING ZHI-CAI, TIAN JING-QI, M.ASIRI ABDULLAH, et al. Two-dimensional hybrid mesoporous $\mathrm{Fe}_{2} \mathrm{O}_{3}$-graphene nanostructures: a highly active and reusable peroxidase mimetic toward rapid, highly sensitive optical detection of glucose. Biosensors and Bioelectronics, 2014, 52(2): 452-457.

[2] ZHANG WEI, HU QI-TU, SU YAN-CHAO, et al. Biocompatible nanostructured poly(xanthurenic acid) $-\mathrm{Fe}_{2} \mathrm{O}_{3}$ /reduced graphene oxide platform for genosensing application. Journal of Electroanalytical Chemistry, 2014, 719(7): 72-76.

[3] WANG MING-YAN, SHEN TAO, WANG MENG, et al. One-pot synthesis of $\alpha-\mathrm{Fe}_{2} \mathrm{O}_{3}$ nanoparticles-decorated reduced graphene oxide for efficient nonenzymatic $\mathrm{H}_{2} \mathrm{O}_{2}$ biosensor. Sensors and Actuators B:Chemical, 2014, 190(1): 645-650.

[4] WANG ZONG-HUA, ZHOU CHENG-FENG, XIA JIAN-FEI, et $a l$. Fabrication and characterization of a triple functionalization of graphene oxide with $\mathrm{Fe}_{3} \mathrm{O}_{4}$, folic acid and doxorubicin as dual-targeted drug nanocarrier. Colloids and Surfaces B:Biointerfaces, 2013, 106(6): 60-65.

[5] YU ZHI-YUAN, LI HE-JUN, LU JIN-HUA, et al. Hydrothermal synthesis of $\mathrm{Fe}_{2} \mathrm{O}_{3} /$ graphene nanocomposite for selective determination of ascorbic acid in the presence of uric acid. Electrochimica Acta, 2015, 158(5): 264-270.

[6] HU MEI-JUAN, JIANG YIN-ZHU, YAN MI. Scalable synthesis of $\mathrm{Fe}_{3} \mathrm{O}_{4} / \mathrm{C}$ composites with enhanced electrochemical performance as anode materials for lithium-ion batteries. Journal of Alloys and Compounds, 2014, 582(1): 563-568.

[7] ZHANG HAI-TAO, ZHANG XIONG, LIN HE, et al. Graphene and maghemite composites based supercapacitors delivering high volumetric capacitance and extraordinary cycling stability. Electrochimica Acta, 2015, 156(4): 70-76.

[8] QUAN HONG-YING, CHENG BAO-CHANG, XIAO YAN-HE, $e$ al. One-pot synthesis of a- $\mathrm{Fe}_{2} \mathrm{O}_{3}$ nanoplates-reduced graphene oxide composites for supercapacitor application. Chemical Engineering Journal, 2016, 286(3): 165-173.

[9] DONG HUI, XU YUN-LONG, JI MAN-DI, et al. High performance of mesoporous $\gamma-\mathrm{Fe}_{2} \mathrm{O}_{3}$ nanoparticle/Ketjen Black composite as anode material for lithium ion batteries. Electrochimica Acta, 2015, 151(1): 118-125.

[10] WANG HUAN-WEN, XU ZI-JIE, YI HUAN, et al. One-step preparation of single-crystalline $\mathrm{Fe}_{2} \mathrm{O}_{3}$ particles/graphene composite hydrogels as high performance anode materials for supercapacitors. Nano Energy, 2014, 7(1): 86-96.

[11] ZHANG LI-LI, YU XIN-XIN, HU HONG-RUI, et al. Facile syn- 
thesis of iron oxides/reduced graphene oxide composites: application for electromagnetic wave absorption at high temperature. Scientific Reports, 2015, 3(19): 1-9.

[12] LI M, CHENG J P, FANG J H, et al. NiAl-layered double hydroxide/reduced graphene oxide composite: microwave-assisted synthesis and supercapacitive properties. Electrochimica Acta, 2014, 134(14): 309-318.

[13] CHENG J P, SHOU Q L, WU J S, et al. Influence of component content on the capacitance of magnetite/reduced graphene oxide composite. Journal of Electroanalytical Chemistry, 2013, 698(11): $1-8$.

[14] LIU GONG-ZONG, JIANG WEI, WANG YANG-PING, et al. One-pot synthesis of $\mathrm{Ag} @ \mathrm{Fe}_{3} \mathrm{O}_{4} /$ reduced graphene oxide composite with excellent electromagnetic absorption properties. Ceramics International, 2015, 41(3): 4982-4988.

[15] ZHU SHEN-MIN, GUO JING-JING, DONG JUN-PING, et al. Sonochemical fabrication of $\mathrm{Fe}_{3} \mathrm{O}_{4}$ nanoparticles on reduced graphene oxide for biosensors. Ultrasonics Sonochemistry, 2013, 20(3): $872-880$.

[16] SUN YOU-YI, ZHANG WEN-HUI, YU HAI-LING, et al. Controlled synthesis various shapes $\mathrm{Fe}_{3} \mathrm{O}_{4}$ decorated reduced graphene oxide applied in the electrochemical detection. Journal of Alloys and Compounds, 2015, 638(14): 182-187.

[17] MAHMOUDIAN M R, ALIAS Y, BASIRUN W J, et al. Synthesis and characterization of $\mathrm{Fe}_{3} \mathrm{O}_{4}$ rose like and spherical/reduced graphene oxide nanosheet composites for lead(II) sensor. Electrochimica Acta, 2015, 169(13): 126-133.

[18] GUO LIANG-QIA, YE PEI-RONG, WANG JING, et al. Three-dimensional $\mathrm{Fe}_{3} \mathrm{O}_{4}$-graphene macroscopic composites for arsenic and arsenate removal. Journal of Hazardous Materials, 2015, 298(21): 28-35.

[19] LIANG JIA-JIE, HUANG YI, OH JI-YOUNG, et al. Electromechanical actuators based on and graphene/ $\mathrm{Fe}_{3} \mathrm{O}_{4}$ hybrid paper. $A d$ vanced Functional Materials, 2011, 21(19): 3778-3784.

[20] WANG HAI-LIANG, CUI LI-FENG, DAI HONG-JIE, et al. $\mathrm{Mn}_{3} \mathrm{O}_{4}$-graphene hybrid as a high-capacity anode material for lithium ion batteries. Am. Chem. Soc., 2010, 132(40): 13978-13980.
[21] XU JUN-MING, WU JIN-SONG, LUO LANG-LI, et al. $\mathrm{Co}_{3} \mathrm{O}_{4}$ nanocubes homogeneously assembled on few-layer graphene for high energy density lithium-ion batteries. Journal of Power Sources, 2015, 274(1): 816-822.

[22] TORU YAMASHITA, PETER HAYES. Analysis of XPS spectra of $\mathrm{Fe}^{2+}$ and $\mathrm{Fe}^{3+}$ ions in oxide materials. Applied Surface Science, 2008, 254(8): 2441-2449.

[23] SONG RUI, FENG SHOU-HUA, WANG HONG-JUN, et al. Effect of organic solvents on particle size of $\mathrm{Mn}_{3} \mathrm{O}_{4}$ nanoparticles synthesized by a solvothermal method. Journal of Solid State Chemistry, 2013, 202(6): 57-60.

[24] KANG YOUNG-SOO, RISBUD SUBHASH, F.RABOLT JOHN, et al. Synthesis and characterization of nanometer-size $\mathrm{Fe}_{3} \mathrm{O}_{4}$ and $\gamma-\mathrm{Fe}_{2} \mathrm{O}_{3}$ particles. Chem. Mater, 1996, 8(9): 2209-2211.

[25] ISABEL PASTORIZA-SANTOS, LUIS M LIZ-MARZAN $\mathrm{N}, \mathrm{N}$-dimethylformamide as a reaction medium for metal nanoparticle synthesis. Advanced Functional Materials, 2009, 19(1): 679-688.

[26] ZHANG ZHONG-JIE, CHEN XIANG-YING, ZHANG XING-FA, et al. Synthesis and magnetic properties of nickel and cobalt nanoparticles obtained in DMF solution. Solid State Communications, 2006, 139(8): 403-405.

[27] WANG YAN,HUANG YI,SONG YOU, et al. Room-temperature ferromagnetism of graphene. Nano Letters, 2009, 9(1): 220-224.

[28] ZHANG WU-XIANG, ZHENG JIAN-ZHONG, SHI JIAN-GU, et al. Nafion covered core-shell structured $\mathrm{Fe}_{3} \mathrm{O}_{4} @$ graphene nanospheres modified electrode for highly selective detection of dopamine. Analytica Chimica Acta, 2015, 853(1): 285-290.

[29] HAZHIR TEYMOURIAN, ABDOLLAH SALIMI, SOMAYEH KHEZRIAN. $\mathrm{Fe}_{3} \mathrm{O}_{4}$ magnetic nanoparticles/reduced graphene oxide nanosheets as a novel electrochemical and bioeletrochemical sensing platform. Biosensors and Bioelectronics, 2013, 49(11): 1-8.

[30] CHEN YU, WANG YONG-LI, ZHANG HAO-BIN,et al. Enhanced electromagnetic interference shielding efficiency of polystyrene/graphene composites with magnetic $\mathrm{Fe}_{3} \mathrm{O}_{4}$ nanoparticles. Carbon, 2015, 82(2): 67-76. 